\title{
Ultra-Fast Flash Observatory: Fast Response Space Missions for Early Time Phase of Gamma Ray Bursts
}

Park, I.H.; Ahmad, S.; Barrillon, P.; Brandt, Søren; Budtz-Jørgensen, Carl; Castro-Tirado, A.J.; Chen, P.; Choi, J.N.; Choi, Y.J.; Connell, P.

Total number of authors:

38

Published in:

E A S Publications Series

Link to article, DOI:

$10.1051 /$ eas $/ 1361083$

Publication date:

2013

Link back to DTU Orbit

Citation (APA):

Park, I. H., Ahmad, S., Barrillon, P., Brandt, S., Budtz-Jørgensen, C., Castro-Tirado, A. J., Chen, P., Choi, J. N., Choi, Y. J., Connell, P., Dagoret-Campagne, S., Eyles, C., Grossan, B., Huang Huang, M-HA., Jung, A., Jeong, S., Kim, J. E., Kim, M. B., Kim, S-W., ... Yashin, I. (2013). Ultra-Fast Flash Observatory: Fast Response Space Missions for Early Time Phase of Gamma Ray Bursts. E A S Publications Series, 61, 501-515.

https://doi.org/10.1051/eas/1361083

\section{General rights}

Copyright and moral rights for the publications made accessible in the public portal are retained by the authors and/or other copyright owners and it is a condition of accessing publications that users recognise and abide by the legal requirements associated with these rights.

- Users may download and print one copy of any publication from the public portal for the purpose of private study or research.

- You may not further distribute the material or use it for any profit-making activity or commercial gain

- You may freely distribute the URL identifying the publication in the public portal 


\section{ULTRA-FAST FLASH OBSERVATORY: FAST RESPONSE SPACE MISSIONS FOR EARLY TIME PHASE OF GAMMA RAY BURSTS}

I.H. Park ${ }^{1}$, S. Ahmad ${ }^{2}$, P. Barrillon ${ }^{2}$, S. Brandt ${ }^{3}$, C. Budtz-Jørgensen ${ }^{3}$, A.J. Castro-Tirado ${ }^{4}$, P. Chen ${ }^{5}$, J.N. Choi ${ }^{11}$, Y.J. Choi ${ }^{6}$, P. Connell ${ }^{7}$, S. Dagoret-Campagne ${ }^{2}$, C. Eyles ${ }^{7}$, B. Grossan ${ }^{8}$, M.-H.A. Huang ${ }^{9}$, A. Jung ${ }^{10}$, S. Jeong ${ }^{10}$, J.E. Kim ${ }^{10}$, M.B. Kim ${ }^{1}$, S.-W. Kim ${ }^{11}$, Y.W. Kim ${ }^{1}$, A.S. Krasnov ${ }^{12}$, J. Lee ${ }^{1}$, H. $\operatorname{Lim}^{1}$, E.V. Linder ${ }^{10,13}$, T.-C. $\mathrm{Liu}^{5}$, K.W. $\mathrm{Min}^{6}$, G.W. $\mathrm{Na}^{10}$, J.W. Nam ${ }^{5}$, M.I. Panasyuk ${ }^{12}$, H.W. Park ${ }^{1}$, J. Ripa ${ }^{1}$, V. Reglero ${ }^{7}$, J.M. Rodrigo ${ }^{7}$, G.F. Smoot ${ }^{10,13}$, S. Svertilov ${ }^{12}$, N. Vedenkin ${ }^{12}$, M.-Z. Wang ${ }^{5}$ and I. Yashin ${ }^{12}$

\footnotetext{
${ }^{1}$ Department of Physics, Sungkyunkwan University, Seobu-ro 2066, Suwonsi, 440-746, Korea

${ }^{2}$ LAL, University of Paris-Sud 11, Orsay, France

${ }^{3}$ National Space Institute Astrophysics, Technical University of Denmark, 2800 Kgs. Lyngby, Denmark

${ }^{4}$ Instituto de Astrofisica de Andalucia (IAA-CSIC), PO Box 03004, 18080 Granada, Spain

${ }^{5}$ Department of Physics, National Taiwan University, 1 Roosevelt Road, Taipei, 106, Taiwan

${ }^{6}$ Korea Advanced Institute of Science and Technology, 291 Daehak-ro, Yuseong-gu, Daejeon 305-701, Korea

7 Universidad de Valencia, GACE, Edif. de Centros de Investigacion, Burjassot, 46100 Valencia, Spain

${ }^{8}$ Space Sciences Laboratory, University of California at Berkeley, USA

${ }^{9}$ Department of Energy Engineering, National United University, 1, Lienda, Miaoli, Taiwan, 36003

10 Department of Physics, Ewha Womans University, 11-1 Daehyun-dong, Seoul 120-750, Korea

11 Department of Astronomy, Yonsei University, 134 Shinchon-dong, Seoul 120-749

12 Skobeltsyn Institute of Nuclear Physics of Lomonosov, Moscow State University, Leninskie Gory, 119234, Russia

13 Institute for the Early Universe, Ewha Womans University, 11-1 Daehyun-dong, Seoul 120-750, Korea
} 


\begin{abstract}
One of the unexplored domains in the study of gamma-ray bursts (GRBs) is the early time phase of the optical light curve. We have proposed Ultra-Fast Flash Observatory (UFFO) to address this question through extraordinary opportunities presented by a series of small space missions. The UFFO is equipped with a fast-response Slewing Mirror Telescope that uses a rapidly moving mirror or mirror array to redirect the optical beam rather than slewing the entire spacecraft or telescope to aim the optical instrument at the GRB position. The UFFO will probe the early optical rise of GRBs with sub-second response, for the first time, opening a completely new frontier in GRB and transient studies. Its fast response measurements of the optical emission of dozens of GRB each year will provide unique probes of the burst mechanism and test the prospect of GRB as a new standard candle, potentially opening up the $\mathrm{z}>10$ universe. We describe the current limit in early photon measurements, the aspects of early photon physics, our soon-to-be-launched UFFO-pathfinder mission, and our next planned mission, the UFFO-100.
\end{abstract}

\title{
1 Introduction
}

In spite of the wide knowledge already acquired about GRBs mainly through CGRO [1], BeppoSAX [2], HETE-2 [3], Integral [4], Swift [5] and Fermi [6], there are still many opened questions about their progenitors and environment. Deeper understanding of GRBs requires not only more statistics of GRBs but also measurements of infrared (IR), polarization, early photons, and high-z GRBs, which can be realized with sensitive IR technology, large volume of crystal for X-ray and large aperture for ultraviolet (UV)/optical/IR, faster response or slewing telescopes, and large aperture with high sensitivity detector, respectively. Moreover, Swift is very unlikely able to extend its operations, much longer than its designed lifetime. Post-Swift missions are foreseen, as well as missions primarily dedicated to high-z GRBs, gamma polarimetry and early photons, i.e. SVOM [7] and JANUS [8], POLAR [9] and UFFO (Ultra-Fast Flash Observatory) series, respectively.

Thorough understanding of GRBs will be aided by multi-wavelength observations in the early emission phase. Hundreds of GRBs UV/optical light curves have been measured since the discovery of optical afterglow [10]. The Swift is the fastest high-sensitivity space observatory that has simultaneously measured X-ray and UV/optical signals in hundreds of GRBs [5]. However, even after nearly 7 years of operation of Swift, the immediate follow-up optical observation of the explosion is scarcely made, because the Swift telescope typically responds in $\sim 100$ seconds. Ground-based telescopes do occasionally respond faster, but only a handful of rapid detections have been made to date with heterogeneous sensitivities. Only a few short duration GRBs have been detected in the UV/optical/IR within the first minute after the gamma ray signal. 
This lack of early observations and the blindness to the rise phase of many GRB optical light curves along with those of other rapidly variable transient sources, leaves many important physical questions arising at the short time scales unexplored. Rapid data collection is also essential for tests of fundamental physics such as constraints on Lorentz violations [11] and CPT [12] from the time delay between different energy photons, or between photons and neutrinos. Coincident or successive observations of the explosion event as an electromagnetic counterpart to a neutrino observatory of gravitational wave observatory signal would greatly improve our understanding of black holes, neutron stars, and strong field gravity.

We have developed methods, for the first time, for reaching sub-minute and sub-second time scales in a spacecraft observatory appropriate for launch on small satellites. We have proposed Slewing Mirror Telescope (SMT) that employs a rapidly moving mirror or mirror array to redirect the optical path at a telescope, instead of slewing the entire spacecraft or telescope to aim the optical instrument at the GRB position. We describe in the following the concept and development of a fast-response optical telescope, the early photon physics with the UFFO project, the current status of the first mission UFFO-pathfinder [13] onboard Lomonosov spacecraft to be launched in 2013, and a proposed full-scale mission of UFFO-100 as the next step.

\section{Current limits of early photon measurements}

The very large field of the Burst Alert Telescope (BAT) of Swift [14] produces a crude sky position via a coded mask technique. The entire observatory spacecraft then slews to point the UV/optical telescope (UVOT) and other instruments at the GRB position. Though the remarkable success of Swift in numerous detections of optical afterglows associated with GRB, only a handful of responses have occurred in less than 60 seconds. The response frequency falls off for response time below $100 \mathrm{sec}$ with an almost complete cutoff by $60 \mathrm{sec}$. Due to finite mission lifetime, Swift is not expected to increase significantly this number of sub-minute responses.

The Swift broadcasts the position of GRB within $5 \sim 7$ seconds to groundbased observatories via the gamma-ray coordinate network (GCN). Although the response of some robotic telescopes on ground (to name a few: ROTSE-I-III [15], RAPTOR [16], PAIRITEL [17], Super-LOTIS [18], BOOTES [19]) is extremely rapid, e.g. $25 \mathrm{sec}$ for ROTSE-III, the sensitivity is far less than that of the Swift UVOT. Due to their small size, and to the limitations of ground-based observing including daytime and weather, together these instruments have managed only a handful of rapid detections [20]. A concurrent optical and gamma observation of the prompt phase of GRB080319B [21] was achieved luckily by TORTORA [22] on REM telescope and by "Pi of the Sky" [23] when this GRB occurred in their field of view and in the field of view of the Konus/Wind instrument [24]. Because of atmospheric scattering or absorption, a $30 \mathrm{~cm}$ aperture space telescope compares favorably in sensitivity to a 4-m ground-based telescope [25]. The slower slew times 
of such larger terrestrial telescopes makes them uncompetitive for the sub-1000 sec regime. The Swift limit of $60 \mathrm{sec}$ response is therefore the practical minimum for sensitive UV/optical GRB studies for the near to mid-term future.

Figure 1 shows the domain of frequency and time accessible by space and ground experiments. The UFFO missions will explore the blank parameter space, the fast- and ultra-fast regimes below $60 \mathrm{sec}$ and even below $1 \mathrm{sec}$, in a systematic survey and thus significantly enlarge the sample of such observations.

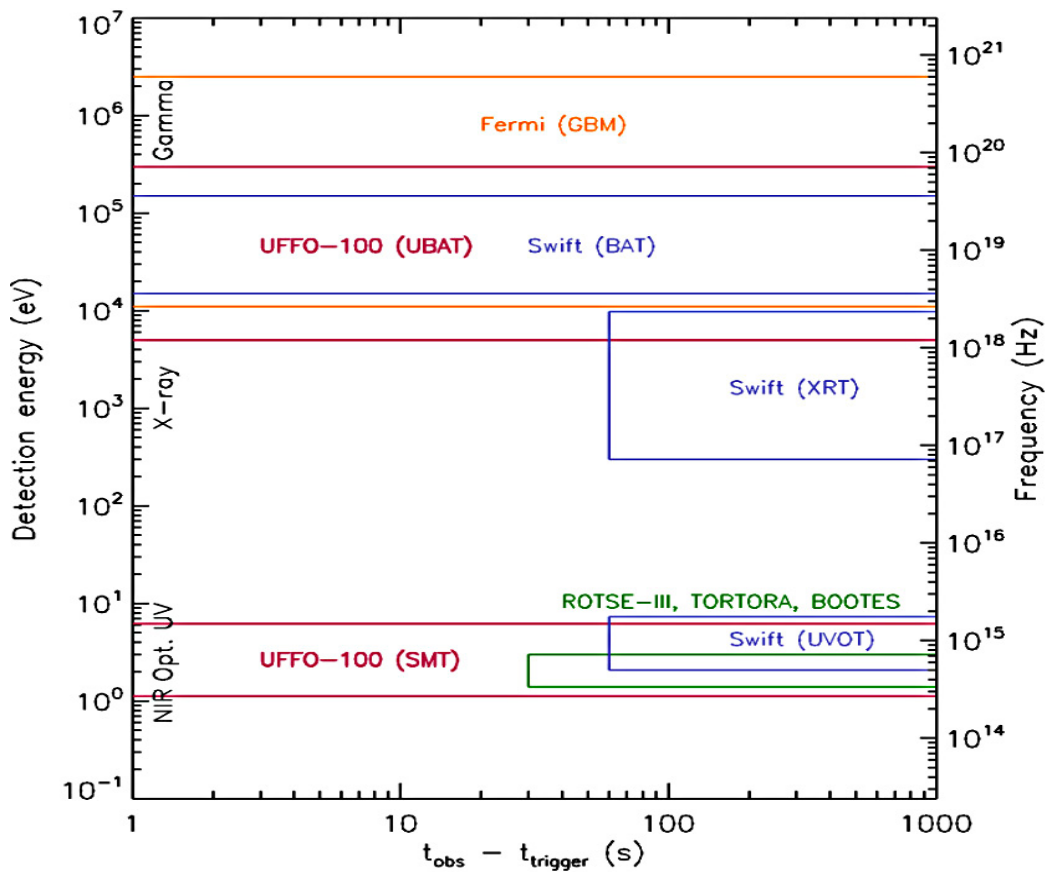

Fig. 1. The accessible frequency and time domain. The UFFO missions will make a systematic survey of the fast- and ultra-fast regimes below $60 \mathrm{sec}$ and below $1 \mathrm{sec}$, respectively.

\section{Slewing mirror telescope}

On localizing or identifying GRB, conventional GRB observatories in space or on the ground must reorient their entire spacecraft or telescope to aim their narrow field instruments at the GRB. Our approach to accelerate the slew capabilities is to redirect the optical path at an astronomical telescope via a substantially more lightweight slewing mirror rather than move the entire payload or telescope [39]. The slewing system can be either a flat mirror or mirror arrays such as MEMS (Micro-Electro-Mechanical Systems) mirror array (MMA), mounted on a gimbal 
platform. In either case, a large field of view (FOV) is accessible without the aberration inherent in wide-field optical systems.

Figure 2 illustrates the concept of SMT. The parallel rays from the distant source are directed on-axis with respect to the fixed optics by the moving mirror system. The net effect is to steer the UV/optical instrument beam, instead of moving the telescope or the spacecraft itself. The beam can be steered by two-axis rotation of the mirror plate, rotation of the individual MMA devices, or rotation of MMA and also gimbal afterward.

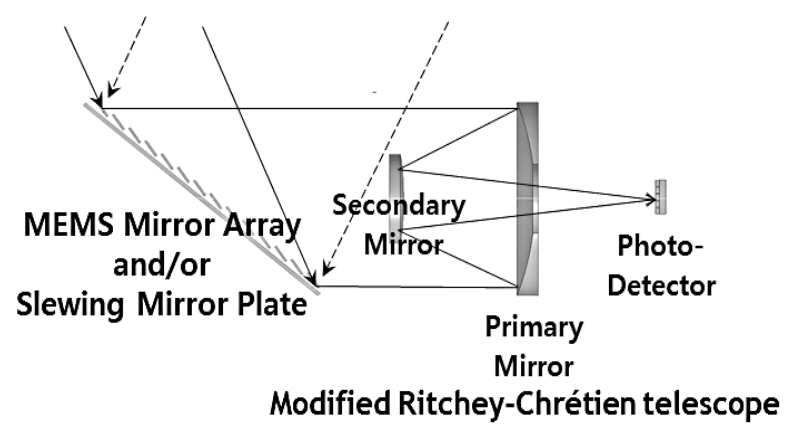

Fig. 2. Schematic of Slewing Mirror Telescope's beam re-direction system.

We find that various types of rotating mirrors move across the entire field of view wider than $180^{\circ} \times 180^{\circ}$, point, and settle in less than 1 sec. In order to build a telescope with milliseconds slew speed both for $\mathrm{x}$ - and $\mathrm{y}$-directions at a time, our lab consortium has produced small mirror arrays driven by MEMS devices. Resembling mirror segments mounted on two-axis gimbals, MEMS micromirrors are fabricated in arrays using advanced silicon and integrated circuit technologies. These MEMS mirror arrays, fabricated like other microelectronics devices, can move, point, and settle in less than a few msec with rotation angle $\pm 15^{\circ}$ off axis and thus FOV of $60^{\circ} \times 60^{\circ}$. Only voltages are applied to tiny electric actuators for rapid pointing to observe bursts. These are extremely lightweight and low power devices that are well suited to the platform of a microsatellite. A series of small prototype MMA system have been developed in our group since 2004 [26]. We fabricated a small prototype of $3 \mathrm{~mm}$ caliber telescope to demonstrate the idea of fast slewing or tracking [27]. It was flown once in space on the ISS in 2008, and once on Tatiana-2 satellite in 2009, with excellent performance, both for nadir observation of transient luminous events occurring in the upper atmosphere [28].

\section{Physics from prompt response UV/optical observations}

Beyond the possible physics with GRBs, the SMT offers a unique opportunity to probe a new, very early emission parameter space to thoroughly investigate the 
rise phase of GRB, which thus far has been observed only occasionally. A variety of rise time physics is as follows.

\subsection{Early rise of light curves}

The discovery of optical afterglows of GRB was a monumental event in modern astrophysics [29], ending the thirty-year mystery of the GRB distance scale. The study of GRB UV/optical afterglows and their host galaxies has led to knowledge of the origin of some types of GRB and the discovery of the most distant explosions known (e.g. GRB 090423 at $\mathrm{z}=8.2$ ) [30]. Much progress has been made in GRB science since the launch of the Swift observatory in 2004 [5]. The observations from Swift did not produce a simple picture of GRB, but rather documented the richness and complexity of this phenomenon. After some $370 \mathrm{UV} /$ optical observations by Swift UV/optical telescope made, a huge variation in light curves has been observed, especially in the early rise time. There appear to be distinct classes of fast-rising (tpeak $<102 \mathrm{sec}$ ) and slow-rising bursts [31]. Additionally, the light curves are complex, with decays, plateaus, changes in slope, and other features that are not yet understood.

It is claimed [31] that among the population of GRB with fast-rising optical light curves, the optical luminosity correlates with the rise time, giving promise as a kind of "calibrated standard candle" much like Type Ia supernovae which would make GRBs useful as a cosmological probe of the very high redshift universe. In order to move this possibility to the status of a refined tool, a larger sample of such optically fast-rising GRBs is required, and in particular, better time resolution is required early on. Fastest-rising bursts often have none or just one measurement in their rising phase - hardly enough to understand the physics in this regime and many other bursts have no early measurements at all. Less than $10 \mathrm{GRBs}$ in this study were measured at less than $100 \mathrm{sec}$ after their burst trigger and not a single measurement was made at less than $15 \mathrm{sec}$ after trigger.

In this respect, several fundamental questions arise. Are there more features in the early light curve that have been missed by such sparse sampling? Does any feature of the rise correlate with the luminosity or a particular aspect of the physics? How many bursts are misclassified because the rapid rise was missed? The need for earlier measurements (faster UV/optical response after the initial gamma-ray burst) is clear and compelling.

\subsection{Short duration GRBs}

GRBs have a separation on the spectral hardness vs. duration plane, and can be classified into short and hard type (SHGRBs) and long and soft type (LSGRBs), according to the duration around $2 \mathrm{sec}[32]$. The short time scale of SHGRB emission, the associated lower luminosity and shorter time scale of the X-ray and optical afterglow lead to speculation that the two classes have fundamentally different physical origins [33]. LSGRBs are thought to originate from the collapse of massive stars, e.g. the collapsar model [34], and SHGRBs from the merger of 
compact objects like neutron stars and black holes (for a review of SHGRBs, see e.g. [35]). Other types of classifications, including those with more of a physical than phenomenological motivation, have been proposed (e.g. [36]). Very short GRB (VSGRB) may originate from the evaporating Primordial Black Holes [37].

The recent progress in SHGRBs is extremely exciting. As of this writing, however, only about $\sim 20$ SHGRBs have had UV/optical measurements often with only one measurement above background, and thus suffer from poor time resolution in their light curves. Two measurements during the decay period are required to determine the most rudimentary decay time constant, assuming a power-law decay. The rise phase of SHGRB optical emission is not observed in most cases. What is the shape of the rise? Is the shape homogeneous? The rise time may give rich information including the size of the system and the surrounding environment. The physical origin of this type of burst remains an outstanding mystery, so any hints as to this origin would be extremely valuable. Is there any prompt UV/optical emission from such events? What would we see if we observed more of these events in the sub-minute or sub-second regime? Are there ultra-short events on the accretion disk dynamical time scale of compact objects (that are beamed so we can see them)? Earlier observations would answer these questions and open a new window probing compact object structure, populations, and evolution.

\subsection{Dark GRBs}

Dark GRBs are those that stand out as having a very faint optical signal compared to X-ray afterglow. Only recently, extinction has been found to be the dominant source of dark GRBs [38]. An alternative scenario, however, suggests that some dark GRBs are simply faded out faster for optical than X-ray emission [39]. In this scenario, the optical emission fades in less than $\sim 102 \mathrm{sec}$, so that most observations would not detect the optical afterglow. Better short time scale observations would shed light on this two-mechanism model.

\subsection{Physical time scales in compact objects}

In a more general sense, resolving the light curve peak time at any epoch gives a hint of the most important physical processes in that epoch. Coalescence of neutron star and black hole systems are features of a number of GRB models, particularly models for the less understood short GRB. The light crossing time of outer accretion disk bounds, the dynamic time scales of large accretion disk systems, and other time scales are in the sub-minute regime, requiring rapid response for their measurement. The time scales of jet formation or deceleration in these smaller systems may also be in this time regime.

\subsection{Emission processes by cross-correlations}

Another general tool that rapid-response observations afford is the correlation of light curves from different bands. If complex light curves in different bands have a 
clear correlation, this is a very strong argument for a physical linkage between the processes of emission in the two wave bands. The delay between the light curves gives further information about both processes [21]. Referring to the correlation of rapid-response light curves accidentally observed earlier, it is intriguing that early UV/optical light curves show good correlation to their $\mathrm{X} / \gamma$ light curves (GRB 041219 [40], GRB 080319B [21]), yet others do not (GRB 990123 [40]). Is this a clue to additional processes, or a hint that the origin of these GRBs is quite different, i.e. SN Ia vs. Ib? What will we see if we can extend these correlations of early emission to SHGRB?

\subsection{Emission processes by spectral slope}

The broad-band spectra of GRBs can be modeled by power laws evolving in time. Chromatic and achromatic jet breaks are important predictions/distinguishing features of models. One feature is the well-known transition from relativistic to non-relativistic emission, the transition from "prompt" emission to afterglow. The change in spectral slope, and the time of this change, are therefore important diagnostics of the interaction of the jet and the surrounding medium, and/or injection of additional energy into the jet. The broad-band spectral slope itself is a discriminator of the electron energy distribution, magnetic field, and other features of the emission mechanism.

\subsection{Test of shock models with bulk lorentz factor}

Measurement of early UV/optical emission can serve as a probe of the physical conditions in the GRB fireball at short times. A simple, nearly model-independent argument [41] shows that the bulk Lorentz factor depends on the time of the early UV/optical emission peak. Measurement of the peak will therefore provide a measurement of the bulk Lorentz factor.

\subsection{Identification of internal shock via fast variability}

Currently, UV/optical emission at early times in typical bursts is believed to come from external shocks, and predicted to have a smooth, monotonic rise (e.g. see [42] and references therein). Observation of an early time UV/optical light curve that more closely resembles a gamma-X light curve, jagged, and with multiple peaks, would clearly indicate the presence of prompt optical emission produced by internal shock. Sub-minute measurements would be required to learn more about such prompt emission.

\subsection{Multi-messenger and fundamental physics}

The coming generation of gravitational wave observatories should regularly detect the coalescence of binary compact systems, the favored scenario for SHGRBs. 
This will open an entirely new field of astronomy. Because of its novel nature, corresponding UV/optical measurements will be highly important to interpret the astrophysics of the event. Moreover, while gravitational wave signals from binary system inspirals have the potential to yield highly accurate distance measurements, they alone cannot break the degeneracy in parameters to yield the redshift - this requires observation of an electromagnetic counterpart such as the GRB. Fast-response optical observations can test Lorentz violations from the time delay between different energy photons, or between photons and neutrinos or early emission with GW. Such a fast-response would be essential for deep understanding of compact objects and cosmology [43].

\section{The UFFO program}

The UFFO will respond to initial photons within a fraction of a second, the hitherto unexplored time domain after the burst of GRB by using the concept of SMT's fast or ultra-fast slewing mirror technology. The UFFO project will be carried out in a series of relatively light payloads to be accommodated readily to micro or small satellites. The first is UFFO-pathfinder that will be flown aboard the Lomonosov spacecraft in 2013. Though the pathfinder is a small and limited, it could be the observational cornerstone of future mission development for rapid responses. The next upgrade version, UFFO-100 with its payload mass of $120 \mathrm{~kg}$ and $40 \mathrm{~cm}$ telescope aperture, is expected to launch in 2018. The UFFO-100 will extend its measurement capability to near-IR (NIR) using dichroic beam splitter on the SMT optics bench. We will demonstrate that such a small mass payload is useful to make major advances in GRB science.

\subsection{UFFO-pathfinder}

The main constraints for inclusion in Lomonosov are $20 \mathrm{~kg}$ total instrument mass and $800 \mathrm{~cm}$ maximum length. Therefore, the system of the UFFO-pathfinder was designed to (i) fit the constraints of the Lomonosov spacecraft, (ii) use all preproven technologies and (iii) to be available for fast delivery. The payload consists of two instruments: SMT for rapid coverage of UV/optical sky and UBAT (UFFO Burst Alert and Trigger Telescope) for X-ray triggers and GRB localization. We have designed a small telescope to provide imaging measurements using a gimbal beam-steering system in SMT described above. UBAT is a wide-field coded mask camera similar to that of the Swift BAT scaled to fit the available mass and size requirements.

The UFFO-pathfinder has passed space environments test, including thermal, vacuum, shock, and vibrations, successfully at National Space Organization of Taiwan (NSPO) in August 2011. The final integration of the flight model to the Lomonosov spacecraft and space environments test is currently under way at a branch of Roscosmos (see Fig. 3). 


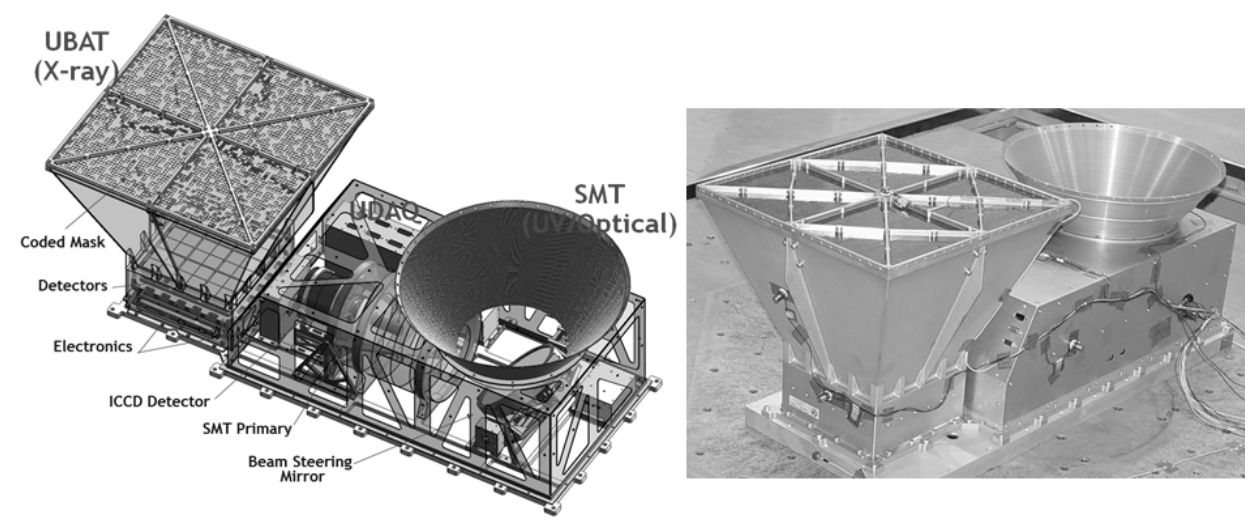

Fig. 3. A rendering of integrated UFFO Pathfinder (left) and fabricated flight model (right).

As a subsystem of UFFO-pathfinder, the SMT is designed for fast observation of the prompt UV/optical photons from GRBs. The SMT/UFFO-pathfinder uses a gimbal system which provides $1 \mathrm{sec}$ response over the entire FOV of UBAT, $90.2^{\circ} \times 90.2^{\circ}$. Electric motors driving gimbal-mounted mirrors are a fundamentally simple and robust technology. For UFFO-pathfinder we used off-the shelf encoders and motors and have already obtained sub-arcsecond settling over $+/-90$ degrees with $\mathrm{t}<1$ sec travel + settle time. The SMT optics includes a Ritchey-Chretien telescope with a $100 \mathrm{~mm}$ diameter aperture. Its field of view is $17 \times 17 \mathrm{arcmin}^{2}$. The focal detector is an Intensified Charge-Coupled Device (ICCD) with a pixel size of $4 \times 4 \operatorname{arcsec}^{2}$ and a wavelength sensitive to $200 \sim 650 \mathrm{~nm}$. The ICCD operates in photon counting mode and could observe faint objects up to 19 magnitude B-star in white light per $100 \mathrm{sec}$, assuming the same performance as Swift and the background estimated by Swift. The SMT has the readout rate of $20 \mathrm{msec}$ and can take 50 frames per second. Two identical flight models of SMT have been built and delivered. The details of SMT can be found in [44].

Numerous instruments have used coded-mask aperture shadow cameras (e.g. BATSE/CGRO [27], BeppoSAX [28], HETE-2 [29], Integral [30], and Swift [31]) to determine positions of GRBs. With the time constraint to meet the launch schedule as well as mass and power constraints for UBAT (only approximately $10 \mathrm{~kg}$ and $10 \mathrm{~W}$ ), we adopted a well-established coded-mask technique similar to Swift BAT but scaled down for the localization of bright, transient X-ray sources. In order to respond over a wider energy range, e.g. $15 \sim 150 \mathrm{keV}$, however, we used pixellated YSO scintillating crystal red out by 36 64-ch multi-anode photomultiplier tubes (MAPMTs) with 36 64-ch SPACIROC ASICs. With only $191 \mathrm{~cm}^{2}$ of detecting area, our collaboration has made a viable camera with which we expect to detect dozens of GRBs per year. The details of UBAT are described in $[45]$. 
The rate of burst trigger depends on the fluence of X-rays. The X-ray flux of typical burst would be one per sec per $\mathrm{cm}^{2}$, while backgrounds are two or three per sec per $\mathrm{cm}^{2}$. Swift can trigger (rate trigger) GRBs typically with $50 \mathrm{msec}$ of X-rays collection. The UBAT detection area is smaller than BAT by factor 25 , so it needs 25 times longer collection time for the same burst. The UBAT will require longer collection time up to $64 \mathrm{sec}$, depending on the brightness of GRBs. Therefore, our estimate is to observe $60 \%$ of BAT bursts typically with about 1.5 sec of collection time, if the orbit is same. On the other hand, larger detection area improves the localization accuracy, i.e. BAT can localize bursts at $90 \%$ probability to a region $1 \sim 4$ arcmin. The UBAT will be able to localize bursts at the confidence level of $7 \sigma$ to a region 10 arcmin across, thus contained fully within the FOV of the SMT. It is noted that it takes only less than a second to determine the position of GRB, using a dedicated FPGA (Field Programmable Gate Array) for "imaging trigger".

The UFFO Data Acquisition (UDAQ) is in charge of central control of the payload not only with preset commands but upload commands from the ground; interfacing to the spacecraft; data collection from SMT and UBAT, storing in several NOR flash memories and transfer to the spacecraft. It is also responsible for monitoring of all housekeeping parameters; calculation of the orbit and recognition of day and night with its photosensors; arbitration and prioritization of triggers from UBAT and BDRG (another Fermi-like payload of Lomonosov); power management, etc.. All of these functions are implemented in an ACTEL FPGA for the low power consumption and fast real-time processing. As mentioned, trigger calculations with the data from UBAT, including rate trigger and imaging trigger, are also performed in another ACTEL FPGA, which reduces the latency significantly, e.g. below 1 sec.

\subsection{UFFO-100}

Awaiting the completion and launch of the UFFO-pathfinder, the UFFO collaboration has been exploring its next step, a more ambitious project: UFFO-100 (named indicating the mass of payload), based on the same design principle but with total mass larger than $100 \mathrm{~kg}$.

The great instrumental challenge of the UFFO concept is to see changes in the optical light curve on short time scales, which requires short exposures. Therefore, the aperture size of the instrument is the fundamental limitation on both the total number of GRB that may be detected, and the time resolution. GRB gamma-ray light curves, even the longer-duration class, have high amplitude variability at every observed time scale. Comparison of the variability between the gamma-X bands and the optical bands can tell us a great deal about the emission physics at the source. Thus far, with the most rapid optical measurements available, it is not known whether gamma-X and optical emission correlates, has lags, or perhaps correlates only in certain types of bursts. There is simply not enough 
short time scale data. The UFFO-100 will answer such an intriguing question of "What would Swift have seen if it could have responded faster?", with the slewing mirror telescope of an aperture $40 \mathrm{~cm}$ as large as that of Swift, but with several enhancements to make it even more sensitive and productive, enabling detections at even shorter time resolution.

Though some enhancements may be restricted by the precise restrictions of payload, UFFO-100 would afford a $1024 \mathrm{~cm}^{2}$ X-ray camera but improved detector technology. The goal is to finally integrate the MMA technology with the motorized slewing mirror and to add a NIR-sensitive camera and specific optical instrumentation to detect the distinguished bursts. The UV/optical and NIR cameras, both with 17 arcmin fields, use the incoming beam from the SMT after being split from a dichroic. Much of the instrumentation, particularly the electronics, will be built on the heritage of UFFO-pathfinder. The pathfinder basic telescope design, fast-mode beam steering, spacecraft bus interface, and data acquisition system architecture will be shared with UFFO-100. We expect UFFO-100. to be flown as one of the scientific payloads of the Russian Resurs-P3 satellite in 2018. The comparison of two payloads performance together with Swift is shown in Table 1.

\section{Summary}

We propose two space missions implementing the UFFO approach in order to investigate a new area of gamma-ray burst phase space both quantitatively and qualitatively. The UFFO equipped with SMT has an extraordinary capability by permitting the first ever systematic study of GRB UV/optical/NIR emission, for example 1 sec after trigger for UFFO-pathfinder and far earlier than 1 sec after trigger for UFFO-100. Our fundamental science objective is to use our ability to probe this new, very early emission parameter space to make measurements of and thoroughly investigate the rise phase of GRB, which is thus far only occasionally observed. In the time domain, this improves on Swift's response by several orders of magnitude. In the spectral domain, we will improve on Swift's sensitivity by $\sim 2.5 \mathrm{mag}$ (assuming the power-law light curve extends at very early times), and we expect to detect afterglow components that are invisible to Swift because of extinction.

The UFFO-pathfinder has now entered the final stage of completion, heading for launch onboard Lomonosov satellite in 2013. The pathfinder is a small and limited, yet remarkably powerful micro-observatory for rapid optical response within $1 \mathrm{sec}$ after X-ray trigger to bright gamma-ray bursts. Its sub-minute measurements of the optical emission of dozens of GRB each year will result in a more rigorous test of current internal shock models, probe the extremes of bulk Lorentz factors, provide the first early and detailed measurements of fast-rise GRB optical light curves, and possibly test the prospect of GRB as extreme $z$ cosmological probes. We foresee not only its exciting findings but the proof-of-principle of this new approach for future GRB telescopes. 
Table 1. Major parameters and expected performance of UFFO payloads together with Swift.

\begin{tabular}{|c|c|c|c|c|}
\hline & $\begin{array}{l}\text { Parameter or } \\
\text { performance }\end{array}$ & UFFO-pathfinder & UFFO-100 & Swift \\
\hline \multirow{8}{*}{ X-ray } & Detector & $\begin{array}{l}\text { YSO crystal + } \\
\text { MAPMT }\end{array}$ & $\begin{array}{l}\text { (Silicon strip) } \\
\text { and (Crystal }+ \\
\text { MAPMT or SiPM) }\end{array}$ & $\mathrm{CdZnTe}$ \\
\hline & $\begin{array}{l}\text { UBAT Field of } \\
\text { View } \\
\text { (half coded) }\end{array}$ & $\begin{array}{l}90.2 \times 90.2 \text { degree }^{2} \\
(1.8 \mathrm{sr})\end{array}$ & $\begin{array}{l}90.2 \times 90.2 \text { degree }^{2} \\
(1.8 \mathrm{sr})\end{array}$ & $\begin{array}{l}100 \times 60 \text { degree } 2 \\
(1.4 \mathrm{sr})\end{array}$ \\
\hline & $\begin{array}{l}\text { X-ray } \\
\text { detection area }\end{array}$ & $191 \mathrm{~cm}^{2}$ & $1024 \mathrm{~cm}^{2}$ & $5240 \mathrm{~cm}^{2}$ \\
\hline & $\begin{array}{l}\text { X-ray } \\
\text { detection } \\
\text { element }\end{array}$ & $48 \times 48$ pixels & $64 \times 64$ pixels & $256 \times 128$ \\
\hline & $\begin{array}{l}\text { X-ray } \\
\text { pixel size }\end{array}$ & $2.8 \times 2.8 \mathrm{~mm}^{2}$ & $2 \times 2 \mathrm{~mm}^{2}$ & $4 \times 4 \mathrm{~mm}^{2}$ \\
\hline & $\begin{array}{l}\text { X-ray } \\
\text { sensitivity }\end{array}$ & $15-150 \mathrm{keV}$ & $5-300 \mathrm{keV}$ & $15-150 \mathrm{keV}$ \\
\hline & $\begin{array}{l}\text { GRB } \\
\text { localization } \\
\text { error }\end{array}$ & $10 \operatorname{arcmin}$ & 4 arcmin & $1 \sim 4 \operatorname{arcmin}$ \\
\hline & $\begin{array}{l}\text { X-ray } \\
\text { collection } \\
\text { time/ GRB } \\
\text { position } \\
\text { calculation } \\
\text { time }\end{array}$ & $1-64 \mathrm{sec} / 1 \mathrm{sec}$ & $1-64 \mathrm{sec} / 1 \mathrm{sec}$ & $\begin{array}{l}1-64 \sec / 5-7 \\
\sec \end{array}$ \\
\hline \multirow[t]{7}{*}{$\begin{array}{l}\text { UV/optical/ } \\
\text { NIR }\end{array}$} & Telescope type & $\begin{array}{l}\text { Ritchey-Chrétien } \\
\text { + Slewing mirror }\end{array}$ & $\begin{array}{l}\text { Modified Ritchey- } \\
\text { Chrétien + Slewing } \\
\text { mirror }\end{array}$ & $\begin{array}{l}\text { Modified } \\
\text { Ritchey- } \\
\text { Chrétien } \\
\end{array}$ \\
\hline & $\begin{array}{l}\text { Telescope } \\
\text { Aperture }\end{array}$ & $10 \mathrm{~cm}$ & $40 \mathrm{~cm}$ & $30 \mathrm{~cm}$ \\
\hline & Field of View & $\begin{array}{l}17 \times 17 \operatorname{arcmin}^{2} \\
\text { over } 70 \times 70 \\
\text { degree }^{2}\end{array}$ & $\begin{array}{l}17 \times 17 \\
\operatorname{arcmin}^{2} \text { over } 90 \times \\
90 \text { degree }^{2}\end{array}$ & $17 \times 17 \operatorname{arcmin}^{2}$ \\
\hline & $\begin{array}{l}\text { Wavelength } \\
\text { range }\end{array}$ & $200 \mathrm{~nm}-650 \mathrm{~nm}$ & $200 \mathrm{~nm}-1100 \mathrm{~nm}$ & $170 \mathrm{~nm}-650 \mathrm{~nm}$ \\
\hline & $\begin{array}{l}\text { Number of } \\
\text { pixels }\end{array}$ & $256 \times 256$ & $256 \times 256$ & $256 \times 256$ \\
\hline & $\begin{array}{l}\text { Physical pixel } \\
\text { scale }\end{array}$ & $4 \operatorname{arcsec}$ & 4 arcsec & $4 \operatorname{arcsec}$ \\
\hline & $\begin{array}{l}\text { SMT data } \\
\text { taking start } \\
\text { time after } \\
\text { trigger }\end{array}$ & $1 \mathrm{sec}$ & $1 \mathrm{msec}-1 \mathrm{sec}$ & $\begin{array}{l}40-200 \mathrm{sec}, \\
\text { typically } 80 \mathrm{sec}\end{array}$ \\
\hline
\end{tabular}


This work is supported by Creative Research Initiatives program (RCMST) of MEST/NRF. IP, EL, GS, HL are members of IEU, which is funded by the WCU program (R32-2009-00010130-0). VL and ACT acknowledge the financial support from the Spanish MINECO project AYA 2009-14000-C03-01/ESP, and PC Taiwan's National Science Council Vanguard Program (100-2119-M-002-025), MP Program of development of Lomonosov Moscow State University. We thank Nauchno-issledovatelskij institut elektromehaniki (NIIEM) of Russia and the National Space Organization (NSPO) of Taiwan for their support in the numerous tests of our instruments for space qualification. Our special thanks to Dr. Yang of KRISS and H.K. Lee of Samsung Electronics for their assistance in the design and manufactures SMT mirrors.

\section{References}

[1] Fishman, G.J., 1994, ApJS, 92, 229

[2] Boella, G., Butler, R.C., Perola, G.C., et al., 1997, A\&AS, 122, 299

[3] Ricker, G., Hurley, K., Lamb, D., et al., 2002, ApJ, 571, L127

[4] Winkler, C., Courvoisier, J.-L., Di Cocco, G., et al., 2003, A\&A, 411, L1

[5] Gehrels, N., Chincarini, G., Giommi, P., et al., 2004, ApJ, 611, 1005

[6] Perna, R., Lazzati, D., Fiore, F., et al., 2003, ApJ, 585, 775

Oates, S.R., Page, M.J., Schady, P., et al., 2009, MNRAS, 395, 490

Perley, D.A., Bloom, J.S., Klein, C.R., et al., 2010, MNRAS, 406, 2473

[7] Paul, J., Wei, J., Basa, S., et al., 2011, Comptes Rendus Physique, 12, 298

[8] Roming, P.W.A., Bilen, S.G., Burrows, D.N., et al., 2012, Memorie della Societa Astronomica Supplement, 21, 155

[9] Produit, N., Barao, F., Deluit S., et al., 2005, Nucl. Instr. Meth. A, 550, 616

[10] Costa, E., Frontera, F., Heise, J., et al., 1997, Nature, 387, 783

Kann, D.A., Klose, S., Zhang, B., et al., 2011, ApJ, 734, 96

[11] Ellis, J., Mavromatos, N.E., Nanopoulos, D.V., et al., 2006, AP, 25, 402

[12] Kostelecky, V.A., \& Mewes, M., 2008, ApJ, 689, L1

[13] Park, I.H., Grossan, B., Lim, H., et al., 2009 [arXiv:0912.0773]

[14] Barthelmy, S.D., 2004, Proc. SPIE, 5165, 175

[15] Akerlof, C.W., Kehoe, R.L., McKay, T.A., et al., 2003, PASP, 115, 132

[16] Vestrand, W.T., Borozdin, K.N., Brumby, S.P., et al., 2002, Proc. SPIE, 4845, 126

[17] Bloom, J.S., Starr, D.L., Blake, C.H., et al., 2006, ASP Conf. Ser., 351, 751

[18] Williams, G.G., Park, H.S., Barthelmy, S.D., et al., 2004, AIP Conf. Proc., 727, 723

[19] Jelinek, M., Castro-Tirado, A.J., Postigo, A.U., et al., 2010, Adv. Astron., 432172

[20] Akerlof, C., Balsano, R., Barthelmy, S., et al., 1999, Nature, 398, 400

[21] Racusin, J.L., Karpov, S.V., Sokolowski, M., et al., 2008, Nature, 455, 183

[22] Beskin, G., Bondar, S., Karpov, S., et al.., 2010, Adv. Astron., 171569

[23] Burd, A., Cwiok, M., Czyrkowski, H., et al., 2005, New Astron., 10, 409

[24] Aptekar, R.L., Frederiks, D.D., Golenetskii, S.V., et al., 1995, Space Sci. Rev., 71, 265

[25] Roming, P.W.A., Kennedy, T.E., Mason, K.O., et al., 2005, Space Sci. Rev., 120, 95

[26] Kim, M., Park, J.H., Jeon, J.A., et al., 2009, J. Micromech. Microeng, 19, 035014

[27] Park, J.H., Garipov, G.K., Jeon, J.A., et al., 2008, Opt. Express, 16, 25, 20249 
[28] Nam, S., et al., 2008, Nucl. Instr. Meth. A, 588, 197

Yoo, B.W., Park, J.H., Park, I.H., et al., 2009, Opt. Express, 17, 5, 3370

[29] van Paradijs, J., Groot, P.J., Galama, T., et al., 1997, Nature, 386, 686

[30] Tanvir, N.R., Fox, D.B., Levan, A.J., et al., 2009, Nature, 461, 1254

[31] Panaitescu, A., \& Vestrand, W., 2008, MNRAS, 387, 497

[32] Kouveliotou, C., Meegan, C.A., Fishman, G.J., et al., 1993, ApJ, 413, L101

[33] Zhang, F.-W., Shao, L., Yan, J.-Z., et al., 2012, ApJ, 750, 88

[34] MacFadyen, A.I., \& Woosley, S.E., 1999, ApJ, 524, 262

[35] Nakar, E., 2007, Physics Reports, 442, 166

[36] Norris, J.P., \& Bonnell, J.T., 2006, ApJ, 643, 266

[37] Cline, D.B., Otwinowski, S., Czerny, B., et al., 2011 [arXiv:1105.5363]

[38] Perley, D.A., Cenko, S.B., Bloom, J.S., et al., 2009, AJ, 138, 1690

[39] Groot, P.J., Galama, T.J., Vreeswijk, P.M., et al., 1998, ApJ, 502, L123

[40] Vestrand, W.T., Wozniak, P.R., Wren, J.A., et al., 2005, Nature, 435, 178

[41] Molinari, E., Vergani, S.D., Malesani, D., et al., 2007, A\&A, 469, L13

[42] Piran, T., 2004, Rev. Mod. Phys., 76, 1143

[43] Stoldosky, L., 2000, Phys. Lett. B., 473, 61

[44] Jeong, S., Nam, J.W., Ahn, K.B., et al., 2013, Opt. Express, 21, 2, 2263

[45] Jung, A., Ahmad, S., Ahn, K.B., et al., 2011 [arXiv:1106.3802]

Kim, J.E., Lim, H., Jung, A., et al., 2011 [arXiv:1106.3803]

Na, G.W., Ahn K.B., Choi, H.S., 2011 [arXiv: 1106.3804] 